\title{
Linx
}

Revue des linguistes de l'université Paris X Nanterre

$73 \mid 2016$

Énonciation et marques d'oralité dans l'évolution du français

\section{Jurons et blasphèmes dans quelques textes des XIV et $\mathrm{XV}^{\mathrm{e}}$ siècles : représentations de l'oralité et transgression}

Dominique Lagorgette

\section{OpenEdition}

\section{Journals}

Édition électronique

URL : http://journals.openedition.org/linx/1640

DOI : 10.4000/linx.1640

ISSN : 2118-9692

Éditeur

Presses universitaires de Paris Nanterre

Édition imprimée

Date de publication : 1 septembre 2016

ISSN : 0246-8743

\section{Référence électronique}

Dominique Lagorgette, « Jurons et blasphèmes dans quelques textes des XIV et XV siècles : représentations de l'oralité et transgression », Linx [En ligne], 73 | 2016, mis en ligne le 01 mars 2017, consulté le 19 avril 2019. URL : http://journals.openedition.org/linx/1640; DOI : 10.4000/linx.1640 


\title{
Jurons et blasphèmes dans quelques textes des $\mathrm{XIV}^{\mathrm{e}}$ et $\mathrm{XV}^{\mathrm{e}}$ siècles : représentations de l'oralité et transgression ${ }^{1}$
}

\begin{abstract}
Résumé ${ }^{2}$ :
À partir de l'étude exhaustive des Manieres de langage de 1396, 1399 et 1415, est posée la question des représentations de l'oralité médiévale et en particulier celle de l'étude de la variation diastratique, des critères que l'on peut mettre en place pour l'évaluer et des méthodes pour l'étudier. Le discours mettant en scène des émotions vives semble particulièrement propice à ce type de recherche dans la mesure où les registres traditionnellement classés comme relâchés sont associés à une nécessité expressive que le registre médian, dit standard, ne permet pas d'assouvir avec la même pertinence pragmatique. Ouvrage didactique particulièrement sensible aux registres, dans la mesure où il propose souvent des alternatives dans les formulations, le recueil des Manieres de langage, guide anglo-normand de F.L.E., présente de nombreuses formules de type juron, et une étude comparée avec les items pragmatiquement équivalents dans des textes antérieurs ou contemporains (une quarantaine de fabliaux continentaux et anglo-normands) puis posté rieurs (une douzaine de farces) permet non seulement de mettre en évidence le fonctionnement du juron, non plus blasphématoire, mais marqueur d'assertion, ponctuant et marqueur de discours, sur une synchronie et une diachronie courte dans différentes variétés de français d'oïl, mais aussi de dresser un premier portrait des registres de langue, de leur usage comme marqué socialement et genré, de leur transmission, de leurs représentations littéraires, de leur évolution ou de leur pérennité, et des mécanismes pragmatiques et lexicaux qui les génèrent.
\end{abstract}

\begin{abstract}
:
Based on an exhaustive analysis of the Maniere de langage de 1396, 1399 et 1415, this article questions the medieval representations of orality, in particular regarding the speech registers and taboo words. On a methodological level, which are the criteria and methods relevant to the analysis of diatopic and diastratic variation in swearwords ? Dialogical sequences involving strong emotional discourse seem a rather good field as low speech registers are traditionally associated to a high expressive need, when the "standard", median speech register provides less pragmatic possibilities and does not relate as well to the violence of the feelings. A manual of French as a Foreign Language such as the Anglonorman Manieres de langage provides numerous swearwords, in various contexts, multiplying the types of speakers (men and women, rich and poor). A lexical and pragmatic study of these occurrences is then compared to anterior and posterior literary texts (about 40 fabliaux and 12 farces from northern France and England) in order to describe the emergence of swearwords as assertive and expletive discourse markers rather than blasphemous items.
\end{abstract}

\footnotetext{
1 Nous dédions cet article à la mémoire de David Trotter, notre merveilleux ami et regretté collègue, qui a guidé nos premiers pas vers l'anglo-normand par sa grande bienveillance et son immense érudition.

2 Ce travail a été réalisé dans le cadre du projet de recherche FFI2013-41355-P « Marqueurs pragmatiques et oralité en linguistique historique du français » du Ministerio de Economía y Competitividad, Espagne, (Plan Estatal I+D+i 2013-16).
} 
Quels sont les moyens, lorsque l'on étudie la diachronie de la langue parlée, pour accéder aux données médiévales authentiques ? Si la question semble aller de soi pour les spécialistes modernes, on sait grâce aux nombreuses études de Claire Blanche Benveniste combien il est délicat d'évaluer la performance spontanée des locuteurs dans des états de langue anciens : la médiation de l'écrit est sans cesse le transmetteur permettant la lecture, avec ses nombreux normes et codages spécifiques : un «vrai » dialogue ou monologue, fidèlement transcrit, est tout bonnement illisible pour le non expert. Pour la période médiévale s'ajoute un autre paramètre : l'auteur fait en effet le lien écrit entre des pratiques et usages parlés et une culture n'ayant pas les vernaculaires comme variétés de prestige et qui, de plus, hiérarchise ces variétés (Lodge 1997). Certes, les dialogues appartenant au domaine de l'écrit donnent de nombreux indices sur ce que devait être un échange entre locuteurs, et ce à tous les niveaux d'analyse ; toutefois, il est bien établi désormais que l'on se situe dans le seul monde des représentations. De plus, même dans ce champ, tous les écrits ne se valent pas : un dialogue de théâtre en vers, par exemple, ne saurait avoir le même degré de vraisemblance qu'un dialogue de roman en prose. Enfin, les publics des textes varient selon les occasions, d'un public lettré ou tout au moins cultivé voire bilingue pour les textes de cours, ou plus varié socialement pour les farces ou les fabliaux, par exemple. À partir de ce paramètre, il semble clair que là encore tous les textes n'ont pas les mêmes stratégies rédactionnelles, ce qui a forcément des retombées au niveau linguistique ${ }^{3}$. Les contraintes stylistiques liées à la création nous incitent donc à prendre en compte non seulement la forme mais aussi le genre littéraire ; elles incitent de plus à chercher d'autres données, non littéraires, contenant du discours afin de vérifier les grilles d'analyse établies de longue date pour les textes littéraires.

C'est ainsi que les Manieres de langage, manuels anglo-normands de didactique du Français langue étrangère de la fin du $\mathrm{XIV}^{\mathrm{e}}$ siècle, s'avèrent une source importante : ces trois textes présentent de nombreuses scénettes mettant en situation ce que l'on appellerait aujourd'hui les apprenants et leur proposant des dialogues-types, souvent marqués registralement. Bien entendu, on ne peut imaginer que ces textes rendent compte du français parlé ; toutefois, ils restent une mine d'or en ce qui concerne les interactions verbales ainsi que les degrés de marquage par le locuteur de la diastratie, dans la mesure où de nombreuses séquences présentent différentes réponses, articulées soit par vel sic, soit par ou, alternant tutoiement et vouvoiement, déférence ou désinvolture envers L2, selon les contextes interactionnels. Un certain nombre de scènes de discorde émaillent ces textes, ainsi que de multiples marqueurs d'émotions vives (interjections, jurons ${ }^{4}$ ), et ce sont les marqueurs assignés conventionnellement à l'accomplissement des actes du juron et/ou du blasphème (qu'il soit voulu/reconnu comme tel ou non) qui retiendront notre attention, comme témoignant des degrés de littérarité ou d'authenticité des discours représentés.

Nous partons en effet de l'hypothèse que le discours marginalisé, celui jugé transgressif par la norme sociale majoritaire (tel qu'insultes, blasphèmes, jurons, par exemple), est dans les textes littéraires à la fois très contrôlé, du fait du contenu pragmatique même, et très développé, car met tant en valeur la virtuosité de l'auteur, son aisance à capter l'air du temps, les cris de la rue, mais aussi son audace à jouer avec le feu par le biais de l'euphémie. Au contraire, les textes didactiques montrent un lexique moins élaboré et plus restreint. Nous étudierons donc, d'une part, les trois

3 Claire Blanche Benveniste aurait classé ces types de performances sur une échelle allant de plus ou moins relâché à plus ou moins surveillé.

$4 \quad$ Nous distinguons ces deux catégories, car elles nous paraissent mélanger les niveaux d'analyse : la première décrit la nature grammaticale de certains items, tandis que la seconde, composée de nombreux items appartenant à des catégories multiples et non prédictibles (préposition, nom, déterminant, adverbe voire verbe), est fondée sur l'acte de langage qu'elle produit, comme son nom l'indique, du reste. Le fait de décrire une locution ou un mot par juron implique un calcul pragmatique de la part du locuteur ; c'est un acte de langage qu'il qualifie, et non une partie du discours à laquelle il réfère. Cette distinction nous paraît essentielle, mais n'est pas toujours claire dans la littérature sur le sujet. 
Manieres de langage et, d'autre part, en regard, un corpus de farces et de fabliaux, car ces textes sont riches en scènes violentes et sont très souvent émaillés de jurons. Notre objectif est d'en décrire le fonctionnement et l'articulation en ancien et moyen français, et nous les envisagerons d'abord dans leur dimension sociale, avant d'en examiner les modalités d'usage, le but étant de mieux cerner l'évolution des actes de langage produits par ces items. Se pose en effet la question de savoir quand la valeur transgressive des jurons a fait place dans certains contextes à une valeur expressive, et comment certains syntagmes nominaux ( $\mathrm{SN}$ ) ont eu valeur de ponctuants.

\section{TRANSGRESSION, TABOU ET JEU}

C'est bien dans le cadre d'une étude des actes de langage transgressant la norme sociale de leur groupe dominant qu'il faut en effet situer les énoncés que nous étudierons : ces propos dérogent, choquent ou amusent, selon leurs contextes, mais ils ne peuvent être pensés hors le cadre d'un discours de référence, comportant des règles et des sanctions s'ils font fi desdites règles. La parole performative a donc dans ce cadre un prix, qui se mesure à l'aune de sa ritualisation et de sa répression. Qu'il s'agisse de faire respecter la norme spontanément par les locuteurs en les éduquant à la courtoisie, comme l'illustre le Conte du Graal, ou qu'il faille littéralement remettre à leur place les déviants, comme s'en chargent les autres locuteurs et en particulier les instances pénalement investies de pouvoir (judiciaires et religieuses, pour le moyen âge), dans tous ces cas, la parole est mesurée, soupesée et contrainte. Toutefois, contrainte ou pas, le tabou linguistique qui relaie le tabou social n'en reste pas moins vivace, et comme le soulignait Emile Benveniste ${ }^{5}$, c'est même là une des conditions fondamentales de son existence. Nous verrons donc tout d'abord comment se met en place ce tabou, selon quelles modalités linguistiques et sociales, puis comment le mécanisme de l'euphémie, notamment, le maintient malgré les interdits.

\subsection{Blastengier, jurer, prier : du divin au social}

Le fait qu'un verbe en ancien français, blastengier, ait pu renvoyer tant à ce que nous nommons insulte qu'à ce que nous identifions comme blasphème, soit respectivement l'insulte à la créature et celle à son créateur, montre combien les frontières sont poreuses entre les actes de langage transgressifs. il en va de même avec jurer, qui peut renvoyer à l'acte de langage conventionnel de l'engagement solennel mais aussi à celui de dire un juron ${ }^{6}$, et avec prier, qui peut référer à l'acte de langage de la demande et à celui de l'intercession conventionnalisée auprès d'une divinité. Cette polysémie pragmatique s'étend aux Sn qui accomplissent ces différents actes, et l'on a déjà souligné combien seul le contexte permet d'en calculer la valeur pragmatique (Favret-Saada 1977, Lagorgette 2003, 2013, notamment), puisqu'on ne peut sans lui évaluer ce qu'accomplissent des SN exclamatifs tels que « Par Dieu », «Bon Dieu », ou encore «Viarge » en Québécois. Le fait de nommer la divinité la convoque, l'apostrophe (là encore dans tous les sens du terme), comme le permet du reste la polysémie du verbe appeler, et gare à celui qui accomplira cet acte magique et sacré en vain, d'où le lien que nous opérons entre ces trois actes.

On notera aussi dans le cas du blasphème et du jurement la présence obligatoire d'un tiers qui devra témoigner de cet acte et en devenir le garant si des procédures légales suivent. L'acte de prière, s'il n'est pas accompli en groupe, se contente, lui, de ne postuler que l'allocutaire. En un

(1974 : 255) : «Un certain mot ou nom ne doit pas passer par la bouche. Il est simplement retranché du registre de la langue, effacé de l'usage, il ne doit plus exister. Cependant, c'est là une condition paradoxale du tabou, ce nom doit en même temps continuer d'exister en tant qu'interdit ».

$6 \quad$ Notons au passage les mêmes propriétés polysémiques pour l'anglais to swear, et ce dès le XII ${ }^{\mathrm{e}}$ siècle (ap. Merriem-Webster dictionary) et pour to curse dès la même période (à la fois jurer au sens blasphématoire et vouer aux gémonies ; ibid.). A curse / to curse est d'ailleurs souvent en anglais un synonyme de swearword / to swear (au sens blasphématoire de jurer). Comme en français, et sensiblement à la même période, les termes liés à l'engagement et au jurement ont donc pris les mêmes propriétés sémantiques et pragmatiques. 
mot, l'agression, tout comme le contrat verbal, requièrent pour fonctionner comme tels un public, tandis que la caresse verbale et la demande peuvent se borner à l'intimité du duo.

Dans les deux premiers cas, l'interaction avec la divinité ne suffit pas à valider l'acte, tout au moins aux yeux des humains : si dans les premiers textes on voit bien des ordalies (on pensera à la Chanson de Roland, par exemple), très rapidement, un tiers se chargera de calculer explicitement le sens de l'acte accompli et d'en déterminer les conséquences, rendant au monde la responsabilité et le choix des formes de la vengeance divine. Il n'y a donc pas de blasphème sans une autorité prenant la place de la divinité qu'elle représente dans l'appréciation de la valeur pragmatique des énoncés. De là à penser que celui qui se donne un tel pouvoir usurpe la place de son dieu et de ce fait commet lui-même un blasphème, il n'y a qu'un pas, qui ne sera pas franchi avant la période moderne. Au contraire, comme le souligne Claude Gauvard (1991 : 807), dès l'ordonnance du 7 mai 1397, sous Charles VI, la dénonciation du blasphémateur est rendue obligatoire.

Cette ordonnance entérine la place fondamentale, dans le calcul du sens, du jugement nécessaire à l'accomplissement complet de l'acte. S'il n'est pas dit et décrit comme tel, l'acte a-t-il eu lieu ? A priori, non, tant que les instances n'auront pas statué et analysé l'intention du locuteur, fondamentale pour le droit, mais aussi pour le dogme chrétien (et la théorie des actes de langage). En d'autres termes, dans ces cas complexes du blasphème, du jurement et du serment, la portée illocutoire de l'énoncé ne vaut que si elle a été reconnue comme telle par un tiers et que si la visée du locuteur coïncide avec elle. Les paramètres tendent donc à se multiplier, décuplant avec eux les risques liés à l'usage de la parole se situant aux marges du sacré et, de par leur énonciation, en recalculant toujours les limites. Les textes fondateurs des trois religions du Livre mettent en garde le croyant contre ces usages dangereux de la parole, comme avec la malédiction, le parjure, le mensonge et la cohorte des péchés de la langue qu'ont bien décrits Carla Casagrande et Silvana Vecchio (1991), dont les lois humaines se sont chargées de délimiter les contours, avec des variations très fortes selon les règnes.

Cela dit, la définition même de ce délit de jurement reste floue, et cela se repère, comme le souligne Corinne Leveleux-Texeira (2000:6), dès la première ordonnance qui lui est consacrée :

(1) «Il sera crié par les Villes, par les Foires et par les Marchiez, chascun mois une fois au moins, que nul ne soit si hardy que il jure par aucuns des membres de Dieu, de nostre Dame, ne des Sainz, ne qu'ils fassent chose par maniere de blasme, ne ne dient vilaine parole, ne par maniere de jurer ne autrement qui torne a despit de Dieu, de nostre Dame, ne des sainz. ${ }^{7}$

$\mathrm{Si}$, dans la Bible, c'est le nom de Dieu qui ne doit être prononcé en vain, on voit ici combien ce tabou est étendu à tout usage des noms des entités sacrées pour des actes de langage divers : non seulement le blâme, mais aussi le serment, l'attestation de la vérité ou toute parole évaluée comme négative (avec tout le flou sémantique que comporte « vilaine parole »). Prendre la divinité à témoin des malheurs qu'elle inflige est en quelque sorte reconnu comme l'acte de reproche qu'il est, si l'on s'en tient au dogme (dans la mesure où la créature doit à son Créateur tous les événements qui lui échoient), mais il s'agit alors d'un reproche insultant car le croyant doit s'en remettre au choix de sa divinité sans s'en plaindre. De même, le mensonge étant en soi un péché, toute parole se doit d'être vraie, d'où la futilité d'attester de la vérité des mots en prenant Dieu à témoin.

$\mathrm{Ne}$ pas respecter ces principes religieux trahit une volonté de puissance du locuteur qui peut être inquiétante, comme nous le signalions (Lagorgette 2013). Permettre que l'on prenne la place du démiurge, dans le cas de l'insulte, ou que l'on en remette la volonté et la perfection en cause, dans le cas du blasphème, équivaut à admettre que la créature peut être investie (ou peut se saisir impunément) des pouvoirs du Créateur. L'aveu, la punition puis le repentir deviennent dès lors 
obligatoires pour le déviant ainsi que pour son groupe. Or, le repentir passe par une série d'actions très ritualisées dont le versant essentiel est public, notamment en ce qui concerne la reconnaissance de la faute et son expiation (Payen 1968). Le fait que le blasphème soit public ne peut être lavé que par des actions publiques elles aussi, de la dénonciation à la sanction. Car si l'on interdit, il faut aussi punir : parfois, c'est la parole qui répare la parole (un blasphème sera lavé par des prières), tandis qu'à d'autres moments, ce sera une action sur le corps du blasphémateur (Saint-Louis, par exemple, est connu pour la violence de ses lois) ${ }^{8}$. Dans tous les cas, la punition, si l'on prend la peine de traiter le problème ${ }^{9}$, est exemplaire et doit marquer aussi bien le locuteur que le public. Il se joue en fait dans ces discussions sur la valeur illocutoire des énoncés bien plus que des questions de linguistique ou même de casuistique : c'est tout l'ordre social qui est alors dans la balance, dans la mesure où le système judiciaire, en dernier ressort, repose sur la crainte du châtiment divin ; comme le note joliment Claude Gauvard (ibid.) : «à chacun de défendre son propre «salut » qui est celui de la communauté du royaume ».

Là où l'insulte peut rester privée, on voit que le blasphème n'est pensé que comme un acte public : sa détection vient du public, et la présence dans le même espace du blasphémateur et de son témoin est essentielle et garantit seule l'acte, dans la mesure où l'allocutaire ne se manifeste pas pour valider sa construction. Toutefois, pour qu'il y ait sanction, un second jugement est aussi nécessaire, car dénoncer n'est que la première phase de la validation : il faut ensuite qu'une autorité investie du pouvoir de punir achève le calcul du sens et le qualifie (au sens pénal du terme) pour qu'il soit. Nous avons donc un cas intéressant d'acte de langage qui s'insère dans la même logique que celle du contrat, en fait, dans la mesure où les deux locuteurs impliqués par l'échange (ici, le blasphémateur et sa divinité) ne suffisent pas à en valider les clauses : un témoin et une instance d'autorité sont aussi des conditions de félicité nécessaires pour que l'acte existe en tant que tel. On retrouve alors l'autre versant sémantique et pragmatique de jurer, qui fixe un contrat avec des conditions d'application et de validité.

\subsection{Du serment au juron : dire sans faire en faisant}

Là ne s'arrêtent pas les points communs entre les deux sens de jurer, qui sont, du reste, contemporains (attestés dès le $12^{\mathrm{e}}$ s. par le TLFi). L'étude de C. Leveleux-Texeira (2007) sur l'aspect doctrinal du serment aux $12^{\mathrm{e}}$ et $13^{\mathrm{e}}$ siècles souligne combien cette notion même est complexe et mouvante, dans un contexte canonique religieux qui tout bonnement l'interdit, et l'évolution des commentaires des décrétistes montre clairement une volonté de réduire cet interdit en le normant :

« L'usage de la parole jurée en régime chrétien ressort en effet d'un exercice de morale paradoxale, qui doit avant toute chose neutraliser ou dépasser la radicalité des interdits posés par le corpus scripturaire. (...) Reprenant à son compte la tradition de suspicion inaugurée par l'Ancienne Loi à l'égard de l'engagement juré, le nouveau Testament l'avait considérablement durcie en posant une prohibition de principe : non seulement le parjure devait être évité mais encore le serment se voyait lui-même interdit sous toutes ses formes. (...) Le serment doit s'insérer dans une ascèse de la parole ; il obéit à un principe d'économie, voire de parcimonie du discours. Il ne doit pas davantage être affaire de sentiment ou d'effusion. Le recours au serment, toujours ponctuel, est présumé répondre à un besoin précis, évalué froidement, sans pathos, à la lumière d'un rapport coût/avantages. Le

Voir notamment les travaux de J. Favret-Saada (1977), J.-P. Pichette (1980), N. Huston (1980), D. Vincent (1982), G. Hughes (1991), B. Lauret (1993), A. Cabantous (1998), C. Leveleux-Texeira (2000, 2007, 2008, 2011$)$, D. Lagorgette (2003, 2005a, 2005b).

9 C. Leveleux-Texeira (2008) rappelle en effet que seuls 1 à $2 \%$ des blasphèmes étaient traités au moyen âge, mais qu'ils accompagnaient très souvent d'autres crimes. Elle précise aussi que le blasphème n'est devenu un délit (au sens légal) que sous Henri IV ; il était auparavant plutôt traité comme un péché ou un vice détestable. 
droit canonique en fait un acte réfléchi, engageant, indifférent à la délectation des sens, vide d'affectivité mais pleinement investi de considérations intellectuelles. (...) Qu'elle tende à se substituer à une expression idolâtrique ou qu'elle vise à emporter l'adhésion d'un interlocuteur dubitatif, l'invocation solennelle du nom de Dieu n'a pas seulement pour effet d'exhausser le cadre ordinaire de l'échange linguistique en introduisant du sacré dans le langage ; elle montre aussi à quel point les rapports sociaux sont pétris de croyance. »

Ce rejet du pathos, de l'affectif, du sacré employé trivialement nous paraît faire le lien entre les différents sens de jurer : d'une part, un acte d'engagement aux conséquences graves, sciemment performé dans un cadre conventionnel et, d'autre part, l'usage d'une partie des formules figées de ce rituel pour garantir sa bonne foi, sans autre rapport à un acte de langage solennel que leur forme (comme le souligne le Dictionnaire de Moyen Français (DMF) par son classement, du reste $)^{10}$. Reste enfin un troisième type d'acte, lui aussi décrit par jurer dès le $12^{\mathrm{e}}$ siècle : l'usage de formules référant à la divinité pour lui exprimer son mépris ; il s'agit alors de l'acte de blasphème, dans la mesure où la visée pragmatique est bien l'insulte d'un allocutaire divin. Dans ces trois cas de figure, l'intention du locuteur reste le critère principal pour trancher, de même que de nombreux autres paramètres tels que contexte, posture et intonation.

Qu'entend-on alors par juron ? Si l'on peut regrouper des locutions sous forme de listes, comme pour les insultes (et l'on parlera de " jurons usuels », eux-mêmes tout aussi culturellement encodés), les corpus nous rappellent rapidement que tout mot peut accomplir l'acte voulu, du moment qu'il est pertinent comme tel dans son contexte d'énonciation. À partir de là, les critères pour savoir si l'on a ou non à faire à un juron sont difficiles à cerner : dans la mesure où l'usage des formules d'adresse à la divinité, d'une part, et des formules de promesse, d'autre part, doit être réservé à l'accomplissement des actes auxquels ils sont conventionnellement dédiés et où tout emploi de ces formules hors cadre constitue en soi un blasphème, on peut étendre à de très nombreuses occurrences ce jugement. Toutefois, ce serait oublier le phénomène très courant, dérivant des serments " affectifs », à savoir l'expression d'une émotion vive par une exclamation, sans visée illocutoire autre que sa propre production et qui dépouille de sa valeur rituelle et magique le mot ; on appellerait alors aussi juron l'usage d'une partie des formules figées de ce rituel pour exprimer des émotions vives, incontrôlées, sans autre rapport à un acte de langage solennel que leur forme ${ }^{11}$. Ils seraient dès lors des ponctuants du discours (Vincent 1993).

Cette difficulté définitoire, que nous avons soulignée dans l'ordonnance de 1269, est partagée par toutes les sciences humaines, de l'anthropologie à la linguistique, et elle vient de la plasticité même du rapport entre forme, sens et acte : en effet, de nombreux auteurs se sont penchés sur la question de la parole dérogatoire, et il est toujours difficile de préciser ce que l'on entend par blasphème et juron (tout comme pour l'insulte, leur acte corollaire). Nous avons déjà souligné combien la confusion entre interjection (partie du discours) et juron est importante, nous y ajouterons aussi la catégorie d'explétif, au sens anglo-saxon d'expletive (Wierzbicka 1992 ; Taavitsainen 1997a et b; Jucker et Taavitsainen 2000 ; Gehweiler 2010), qui renvoie à l'expression de l'émotion vive, catégorie elle-même sujette à des sous-catégorisations qui en fait reprennent les trois sens de jurer. Cette dernière fonction, à la fois phatique, expressive et émotive dirait Jakobson, s'accommode de n'importe quel lexème pour opérer, comme l'ont noté tous ces auteurs pour l'anglais, mais aussi, notamment, J.-P. Pichette (1980), D. Vincent (1982) pour le

10 Un film très populaire des années 1990, La vie est un long fleuve tranquille, d'Etienne Chatiliez, semblait concilier cet ensemble lorsque la pieuse Madame Lequenois, d'un « ne jurez pas, Marie-Thérèse, ne jurez pas ! », rebutait sa malheureuse gouvernante, dépassée par les événements et garantissant son honnêteté par un lancinant « mais je vous jure, Madame ».

11 Le TLFi suit ce découpage : «Juron : A. Exclamation offensante à l'égard de Dieu qui traduit une réaction vive de colère, dépit ou surprise (...). B. P. ext., fam. Interjection ou exclamation grossière ou familière qui traduit une réaction vive de colère, dépit ou surprise. Synon. vieilli ou littér. Jurement. (...)» 
québécois, ou encore J. Orr (1957), N. Huston (1980) ou D. Lagorgette (2005b) pour le français hexagonal moderne et contemporain.

Parmi les explétifs, ce que l'on nomme euphémie est l'un des mécanismes rendant possible le contournement du «mauvais serment » : comme le rappelle Paul Zumthor (1953 : 177), « Le grec euphêmia, puis euphêmismos, relève originellement du vocabulaire sacral. Il désigne en principe l'emploi d'expressions fastes au cours du sacrifice ; en pratique, l'exclusion de toute expression néfaste au sein du sanctuaire ». Hors sanctuaire, le fait de déformer les formules rituelles tout en les laissant deviner, par proximité phonétique ou morphologique, permet d'accomplir un acte proche de l'acte prohibé et d'en tirer le bénéfice, sans en prendre les risques, par la transgression au moins symbolique de l'interdit. Il s'agit d'un jeu avec le feu, qui par le biais de la désémantisation finit par perdre toute valeur transgressive, pour ne plus garder que la valeur expressive. Les formes euphémisées doivent être proches des locutions taboues pour pouvoir fonctionner, c'est leur contrainte, et tout nom répondant à ce critère est pertinent, ce qui rend ces items interchangeables (comme «flûte » ou «fichtre » pour « foutre » en français). Le terme de substitution, avec plus ou moins d'ingéniosité créative, remplit l'espace du terme tabou, en reprenant le son initial et parfois même le nombre de syllabes.

Les phénomènes de maquillage sont très divers et font feu de toutes les techniques possibles, allant de modifications phonétiques et morphologiques (comme dans Dieu > bleu, bieu, beu, bé) qui peuvent se cumuler à la modification syntaxique (comme avec morbleu < par la mort de Dieu) à l'emploi de ces mécanismes sur un item métaphorique (cor bœuf < cœur de Dieu). On relève aussi des déplacements métonymiques, comme au Québec où les objets liés au culte sont les référents (tabernacle, hostie, calice). Enfin, la troncation ou la suppression du COD permettent de masquer encore plus le «vilain serment » initial, comme dans jerni (pour je renie Dieu) : ces mécanismes peuvent se cumuler, au point de rendre l'item d'origine méconnaissable. Des phénomènes de figement se mettent graduellement en place et rares ne sont pas en diachronie les éléments grammaticalisés (c'est-à-dire ayant changé, par ces opérations, de nature grammaticale et de fonction syntaxique) et pragmaticalisés, la force illocutoire de blasphème disparaissant au profit de l'expression quasiment réflexe de sentiments ne renvoyant plus au sacré et accomplissant d'autres actes. Dès lors, ces SN nouveaux sont pensés comme des interjections, surtout lorsqu'il est difficile de retrouver leur forme et leur sens initiaux (cf. Gehweiler 2010, par exemple).

D'autres tests ont néanmoins montré aussi que même les formes euphémisées les plus répandues et figées dans les traditions orales locales peuvent être réanalysées, ce qui mène les locuteurs à retrouver assez aisément le $\mathrm{SN}$ initial et son sens premier, et alors, généralement, le tabou fonctionne de nouveau dans la mesure où les locuteurs, et en particulier les locutrices, sont choqués par ces mots (Lagorgette (2005b)). Car les usages des jurons sont marqués non seulement par l'appartenance à une communauté, mais aussi par des paramètres globaux tels qu'âge, classe sociale, zone géographique et genre, et des paramètres individuels. En somme, non seulement le juron est le reflet d'une époque dans une culture donnée, mais il est aussi produit selon des paramètres reliés à tous les types de variation, comme nous l'examinerons maintenant.

\section{REGiSTRES ET VARIATION(S)}

Se pose tout d'abord la question de l'identification des occurrences, puisque les formes ne correspondent pas toujours aux mêmes actes ; il est alors urgent, comme dirait William Labov, de distinguer ce qui est dit de ce qui est fait. Les différents textes du corpus regorgent de locutions référant à la divinité dans des contextes non conventionnellement assignés à cet acte, afin qu'elle prenne sous sa protection le locuteur ou son allocutaire (comme par exemple si m'aist Dieu, Dieu 
mercy, en noun de Dieu) dans des contextes où de telles demandes ne sont pas nécessaires ${ }^{12}$ :

(2) Maniere 1415, p.71 : «Donques, sire, alomes e[n]semble en noun de Dieu et nous y vendrouns en bone temps par le haut solayle, si Dieu plest.»

$\mathrm{Si}$, stricto sensu, ces formules devraient prendre place dans la cohorte des blasphèmes, puisque le divin est mentionné pour des actions aussi banales que le souhait d'une bonne journée à autrui ou encore l'espoir qu'une entreprise se passe pour le mieux, deux éléments (outre le bon sens) nous incitent toutefois à les rejeter comme tels : d'une part, leur haute fréquence et la multiplicité des locuteurs les employant à tout bout de champ, et d'autre part leur visée pragmatique. On a en effet pour beaucoup de ces nombreux énoncés des contextes sans conflit, sans revers de fortune et d'un tel prosaïsme qu'il est inimaginable que les personnages aient voulu blasphémer ; au contraire, les actes de langage accomplis par ces expressions sont de l'ordre de la demande et donnent une tonalité pieuse au discours des locuteurs. Un autre critère est qu'elles ne sont pas des attestations de la vérité de l'énoncé ou de la sincérité de la croyance du locuteur, et encore moins de l'insulte à la divinité. Resterait alors l'expression réflexe d'une émotion vive, automatisme de discours et point commun de cet ensemble de locutions référant au divin « en vain ». Cet aspect explétif n'est toutefois pas un critère suffisant, puisqu'il pourrait marquer la joie, la surprise devant une bonne fortune et accomplir la louange : c'est donc l'acte de langage et lui seul, resitué en contexte, qui permet de trancher entre les jurons et les autres formules renvoyant au sacré.

Formellement, on pourrait s'attendre à ce que des structures soient régulières et permettent d'affiner ce classement : par Dieu s'oppose alors à pour Dieu, le premier relevant du juron et le second de la demande, la préposition permettant d'opposer les deux énoncés et les deux actes. Toutefois, l'euphémie jouant, là aussi le critère s'avère insuffisant seul : ainsi, " Marie », dans la Maniere de 1415 (cf. infra, ex. 6.d), ressemble-t-il à un terme d'adresse banal, mais il peut aussi être l'invocation, sous forme de juron, de la Vierge ; hors contexte, on ne peut savoir si «par» a été tronqué ou s'il s'agit d'un vocatif (succès et efficacité de l'euphémisation). Autre critère, la position dans la phrase : les jurons seraient en tête ou en clôture d'énoncé, voire " autonomes ", en tous cas détachés. Les corpus, s'ils montrent en effet une majorité de cas dans l'une de ces trois configurations, ne limitent pas les jurons dans ces places précises; il s'agit donc d'un critère de plus, mais qui là encore ne peut suffire, d'autant que ces places peuvent elles aussi être remplies par les locutions détachées de demande. Enfin, les choix lexicaux semblent de bons candidats, dans la littérature sur le sujet, pour établir si l'on a affaire ou non à un juron, et c'est en effet pertinent mais pas suffisant, puisque l'euphémie se joue des champs sémantiques, et tire même profit de leur brouillage. C'est donc plutôt l'accumulation et le croisement de cet ensemble de critères qui a gouverné notre relevé. Enfin, nous proposons un test très simple afin de classer ces usages : remplacer la locution contenant « Dieu » par l'une des trois possibilités entrant dans la catégorie globale des jurons, soit respectivement par «Dieu est vain », version développée du tabou religieux initial (= juron blasphématoire), par l'adverbe « sincèrement » (attestation de la sincérité de $\mathrm{L} 1$ = juron assertif) ou par une interjection de type onomatopée (= juron expressif). Si parfois cette dernière substitution fonctionne aussi avec les locutions positives (ce qui est logique, puisque l'expressivité peut être positive ou négative), ce n'est jamais le cas avec les deux précédentes.

Parmi les jurons retenus, nous avons distingué trois catégories lexicales : ceux qui engagent un être humain (le locuteur ou l'allocutaire), ceux qui engagent la divinité, ceux qui engagent son ennemi, le diable, auxquelles s'ajoutent les euphémismes, qui eux-mêmes peuvent s'insérer dans chacune des catégories précédentes. La confrontation des différents textes du corpus permet de mettre en évidence des termes récurrents en diachronie, parfois sans surprise comme avec par

12 Nous excluons donc tous les énoncés où ces mêmes locutions accomplissent des actes de langage de type prière et salutations. 
Dieu, ce qui nous informe sur les usages de l'oralité, mais aussi un grand nombre d'hapax, liés vraisemblablement à la créativité des auteurs et à leur relation aux stéréotypes qu'ils choisissent de représenter. De manière très plausible et attendue ${ }^{13}$, peu d'énoncés appartiennent au blasphème dans l'ensemble des textes, comme nous allons maintenant le détailler. Entre assertif et ponctuant, reste à établir si ces usages étaient perçus comme tels ou comme encore «dangereux » par les locuteurs. Un manuel de didactique représentant des tendances plutôt que des cas particuliers, les Manieres nous paraissent particulièrement intéressantes dans ce cadre.

\subsection{Des jurons à vous damner un saint? L'art et les Manieres}

Les Manières de Langage de 1396, 1399 et 1415 érigent le dialogue comme modalité didactique majeure, mettant en scène des sections de discours direct présentant des alternatives. Rédigées en Angleterre, avec de nombreux traits anglo-normand (Kristol 1995), elles visent à apprendre le «bon français » à leurs élèves et à leur rendre les interactions quotidiennes aisées : cette suite de scénettes dialoguées où un anglophone se renseigne auprès des natifs, marchande, courtise, se dispute, etc., fait la part belle aux registres et à la variation diatopique. Rendant compte de situations aussi variées que possible, les moments où les locuteurs s'enflamment ne manquent pas, qu'il s'agisse de déclarer sa convoitise ou sa colère, et les jurons sont présents dans les trois textes, avec 87 occurrences.

Parmi les locutions qui engagent l'humain, on distinguera d'abord celles référant au locuteur, lequel jure par son corps, son âme, son « serement », marquant ainsi des degrés dans l'assertion de sa bonne foi, de sa sincérité ${ }^{14}$, comme dans cette histoire courtoise qui se greffe dans le dialogue entre un galant et sa belle :

(3) $\mathrm{M} 96^{15}$, p.15 : «"Par mon surement, ma tresdouce dame”, fist il, “je su si disconfiz et pauereux au coer que n'ay je plus d'appetit ne voluntee pur esbatre ovesque vous que un petit enfaunt que n'entent point de male." "

Dans ce cas particulier, il s'agit de discours rapporté, et qui plus est d'un style qui tranche avec les modalités d'énonciation usuelles du couple : c'est l'un des personnages dont on raconte l'histoire qui s'exprime ainsi. Comme en témoigne d'ailleurs le terme d'adresse proche, plus élaboré que les plus usuels du recueil, cette locution empruntée est présentée comme appartenant de plein droit au discours amoureux littéraire - ce que l'on n'attendrait pas forcément parmi les représentations des locuteurs, eu égard au tabou. La locution est donc déjà figée avec cette valeur à ce stade de son évolution, et nous la retrouvons graphiée telle dans la suite de cette histoire ; le fait que ce soit un style littéraire qui soit montré permet aussi de proposer que cet usage de la locution était perçu comme pertinent dans un contexte de langue soutenue. Nous la retrouvons dans les Manieres de 1396 et de 1399 avec la graphie serement ou serment ; la forme « surement » n'est pas attestée ailleurs dans l'Anglo-Norman Online Hub (ANOH), ni dans la Base de Français Médiéval (BFM) et le $\mathrm{DMF}^{16}$. Cette variante marquant un registre, c'est peut-être une hypercorrection ; mais ce pourrait aussi être un euphémisme, voire les deux à la fois, l'une n'excluant pas l'autre.

Parmi les 87 occurrences de jurons (au sens large) des trois Manieres, on relève 21 occurrences engageant L1 (10 dans la Maniere de 1396, 9 dans 1399 et 2 dans 1415).

\footnotetext{
13 On pouvait en effet s'y attendre au vu du peu de condamnations pour ce délit, d'une part, mais aussi parce que le danger lié à cet acte est tel que même les registres criminels se contentent du métadiscours et ne rapportent pas les termes exacts lorsqu'un jugement de blasphème a été rendu (Leveleux-Texeira, 2007, notamment).

$14 \quad$ Voir aussi les exemples (5.a), (5.b).

15 Nous référons dans les exemples par M96 pour Maniere de 1396, M99 pour Maniere de 1399 et M15 pour Manière de 1415.

16 Ces trois précieuses bases sont consultables en ligne et nous ne saurons jamais assez remercier leurs concepteurs : http://www.anglo-norman.net/ ; http://txm.bfm corpus.org/ ; http://www.atilf.fr/dmf/
} 
Le deuxième type d'engagement dont le garant est un être humain concerne cette fois l'allocutaire, et nous n'en relevons que deux exemples, dans la Maniere de 1396 :

(4) M96, p.12: «Par vostre foy, coment vous est il a jour de hui ?» (et p.17-6, cf. ex. (5.a))

Le deuxième type de locution se rapproche formellement du blasphème prototypique, dans la mesure où c'est à la divinité que réfère l'expression, dans la majorité des cas (35 occ. sur 87 ) avec « par Dieu » (voir ex. (6.e) et (16)), mais il arrive aussi que la référence à Dieu soit bien plus violente, puisque sa mort est évoquée :

(5.a) M96, p.17 : «Dit le closier d'un gardin a un fosseour qi foue lez terres ou les fosses in ceste manere : «Mon amye, par ta foy, qu'as tu gaigné ceste sepmaigne?

- Par mon serement, j'en ay gaigné toute ceste sepmaigne pur fouir les terres et faire des fosses bien parfons que .xii. deniers et mes despenses.

- Par la mort du Dieu, c'est bien petit. »»

(5.b) M96, p.38 : «Par la mort Dieu, biau sire, se je eusse volu, je eusse eu huy ou matinee pour mesmes les anes .x. d. Ore me croiez se vous vuillez.

- Il ne vous faudra ja ainsi jurer, car je vous en croi bien a primer mot sanz plus sonner. Ore ditez moy a un mot, que paierai je ?

- Par ma foy, j'en avrai atant pour yceulx ou autrement je n'avrai riens. »

Dans ce dernier exemple, L2, en désaccord avec l'usage des jurons par L1, ne se trompe néanmoins pas sur leur valeur pragmatique d'assertion de la sincérité, comme il le souligne par son commentaire métadiscursif. Il faut dire que L1 ne peut produire le moindre énoncé sans l'émailler d'un juron expressif ou assertif.

Dans cette même catégorie des références à la divinité, on relève aussi des occurrences renvoyant à la Vierge (ex.6.a, c, d) ou à des saints nommés précisément (ex.6.b, c) :

(6.a) M96, p.31 : «Par Nostre Dame, save vostre grace, non fais. Ore alez a Dieu, sir. »

(6.b) Ibid., p.16 : «Par seint Jakes, m’amye, si fist il. »

Dans cet ensemble de 59 occurrences, la divinité évoquée (et non invoquée) est érigée en garante du discours et de la bonne foi, de la sincérité du locuteur. Cette haute instance devient témoin des intentions affichées par L1.

Chaque texte développe son propre réseau divin : en effet, la Maniere de 1415 ne recourt ni aux saints, ni au diable, ne référant qu'à la Vierge et à Dieu, tandis que la Maniere de 1396, lors de la rencontre entre l'apprenant (a priori anglais) et le Français, marque le discours de ce dernier diatopiquement grâce à un juron assertif (test de l'adverbe réussi) :

(6.c) p.33: «Hé, plust a Dieu et à la virgyne Marie, mon tresdoulx amy, que je sceusse si bien et graciousement parler franceys com vous savez, quar veraiment $j$ 'en fuisse donques bien aisee au coer.

- Par Nostre Dame de Clery, je voudroy que vous sceussez, mais toutzvois vous parlez bien assez, ce m'est avys, qar je pense bien que vous avez demurré grant piece la, depuis que vous parlez si bien et plainement la langage.

- Par seint Paul, je n'y fu unques mais. »

On notera que chaque locuteur ouvre son énoncé par un juron (voir aussi 5.a), dans un mécanisme de symétrie que nous avions déjà signalé pour les termes d'adresse (Lagorgette 1998). Il arrive aussi parfois que ce soit un seul locuteur qui recoure à un juron chaque fois différent :

(6.d) M99, p.63 : « - Le roy, coment le fait il ? - Par Dieu, sire, la mercy Dieu, tresbien. -Et la roigne aussi ? - Marie, elle est en bon point, come j'ay ouy. »

Ce type d'usage s'apparente au réflexe verbal (voir aussi 5.b). En (6.d), la symétrie de construction avec la réplique précédente du même locuteur permet d'interpréter le nom propre 
comme juron expressif, en plus des autres critères et du test avec l'adverbe ${ }^{17}$.

Les références à l'Ennemi, au diable, qui représentent 5 occurrences (soit 5,75\%), nous paraissent marquer un degré supérieur dans l'émotion :

(7) M96, p.16 : « Janyn, que faitez vous?

- Mon seignour, se vous plest, je songe.

- Reveille toy, de par le deable et de par sa miere ové tout, ou autrement je toy dorray un tiel

sufflet que tu penseras de moy deci as quatre jours, me croies se tu vuis. »

Dans cet échange, hiérarchiquement construit puisque Janyn est le subordonné de L1, la violence monte graduellement et le juron accompagne la menace d'une agression physique punitive. Nous retrouvons cette même locution deux fois dans ce texte (p.15 et 43), dans les mêmes contextes de conflit et de menace ; la dyade diable et sa mère (calquée sur la même relation filiale chrétienne) paraît renforcer la violence de l'énoncé. Il s'agit d'hapax dans l'ANOH, la BFM et le DMF, et la Maniere de 1399 se contente de «par le diable » (1 occ.), locution relevée aussi dans la Maniere de 1396 (1 occ.).

Comme le note Robert Martin dans la notice «diable » du DMF, ces formules sont « des formules d'invocation, en prenant le diable à témoin, pour garantir ce qui est dit, en partic. l'engagement qui est pris (au risque d'encourir son action maléfique en cas de défaillance) ». Peut-on alors parler d'un blasphème, dans la mesure où c'est l'Ennemi et non la divinité qui est évoquée ? A priori, non. Toutefois, ces formes, en particulier les plus longues, sont bien plus transgressives que les autres jurons des trois textes. Elles s'apparenteraient à des assertifs, d'un degré haut.

Enfin, sur 87 occurrences, seule la Maniere de 1415 présente quelques cas (deux, en fait) de modifications morphologiques et phonétiques portant sur « Dieu» :

(8) M15, p.75-20 : - Dame, pernez vostre hanap, par Diee.» (ibid., p.75-29)

Le texte emploie par ailleurs « Dieu », même s'il limite beaucoup ses usages de jurons (4 en tout) et de références à la divinité en général, respectant visiblement le tabou à la lettre : il est donc probable que c'est bien d'euphémie qu'il est ici question, d'autant qu'il s'agit d'un hapax (le terme n'apparaît nulle part ailleurs dans le corpus de l'ANOH et pas dans le DMF). Ceci renforce notre lecture des jurons comme assertifs ou expressifs et non blasphématoires. L'euphémisation montre aussi que ce sont les formes renvoyant à la divinité, les premières en termes de transgression, qui sont perçues comme les plus dangereuses, les moins susceptibles d'être employées sans conséquences ; le figement semble plus avancé dans ce cas et plus tôt.

Le Tableau 1 récapitule la répartition de l'ensemble des jurons dans les trois Manieres, et il est net que les formules référant à la divinité l'emportent largement dans les usages :

Tableau 1

\begin{tabular}{|c|c|c|c|c|c|c|}
\hline & \multirow{2}{*}{$\begin{array}{c}\text { L1 } \\
(24,2 \%)\end{array}$} & \multirow{2}{*}{$\begin{array}{c}\mathrm{L} 2 \\
(2,3 \%)\end{array}$} & \multicolumn{2}{|c|}{ Divinité $(67,8 \%)$} & \multirow{2}{*}{$\begin{array}{l}\text { Diable } \\
(5,7 \%)\end{array}$} & \multirow{2}{*}{$\begin{array}{c}\text { [Dont } \\
\text { Euphémie }\end{array}$} \\
\hline & & & Dieu & Autres & & \\
\hline $1396(=50)$ & 10 & 2 & 24 & 10 & 4 & 0 \\
\hline $1399(=33)$ & 9 & 0 & 9 & 14 & 1 & 0 \\
\hline $1415(=4)$ & 2 & 0 & 2 & 0 & 0 & 2 \\
\hline Total $(=87)$ & 21 & 2 & 35 & 24 & 5 & $2]^{18}$ \\
\hline
\end{tabular}

17

De plus, ce nom propre employé comme juron explétif est attesté en anglais par Hughes (1991 : 249) comme autrefois courant en Angleterre, pays de rédaction de la Maniere de 1399 ; ce serait donc une variante marquée diatopiquement, dans la mesure où le corpus continental de fabliaux puis de farces ne l'atteste que très rarement : on relève ainsi 9 occurrences dans le DMF, toutes dans des Miracles du $14^{\mathrm{e}}$ s., antérieurs aux Manieres. On notera toutefois que les 5 occurrences de la Maniere de 1399 sont les seules dans les textes de l'ANOH. "Ave Marie », «(par) Sainte Marie ! » et «Vierge Marie » sont en revanche fréquemment attestés dans le corpus continental.

L'euphémie pouvant porter sur l'ensemble des catégories lexicales, nous ne l'avons pas incluse dans nos 
Ces mêmes catégories, ainsi que l'euphémie, sont présentes dans notre corpus littéraire de contrôle (fabliaux continentaux et anglo-normands du $12^{\mathrm{e}}$ au $14^{\mathrm{e}}$ siècles, et farces du $15^{\mathrm{e}} \mathrm{s}$.). Toutefois, si, dans les fabliaux, nous trouvons des références au locuteur (ex.9), à la divinité (ex.10) et des formes euphémisées (ex.11) :

(9) Le chevalier qui fist parler les cons, p.568: «Par mon chief, Huet, tu diz voir » (10.a) Des trois dames qui troverent l'anel, pp.110-112 : "Lors prant li sires a jurer : / «por les sainz Deu, dite[s] vos voir?»

(10.b) D’Auberee, p.526-527 : «Dame, por Deu et por son non, / dist le borgeois, dites vos voir?»

(10.c) Estourmi, p.488 : «Vois ! Por le cul sainte Marie ! / fet Estormi, je sui matez./ »

(11) Trubert, p. $129:$ «Pour le cuer bieu, estes vos fous?»

nous ne relevons aucun référence au diable. En revanche, les quatre types sont présents dans les farces avec des références au locuteur (ex.12), à la divinité (ex.13), au diable (ex.14) et de nombreux termes appartenant à l'euphémie dans ces trois domaines (ex.15) :

(12) Cuvier, vv.17-18 : «Jaquinot : Mais par le sang que Dieu me fist / Je seray maistre en ma maison»

(13) Cuvier, vv. 147-150 : « Jaquinot : Rien n'en sera, par le bon saulveur ! / Cinq ou six fois ! Vertu sainct George ! / Cinq ou six fois ! ne deux ne trois !»

(14) Cuvier, v.193 : «non ferez, non, de par le dyable !»

(15.a) Chaudr., v.54-55: « L'homme : Sainct Mort, voicy dure passion ! / Par sainct Copin, je suis tanné. »;

(15.b) Cuvier, v.153-154 : «Jaquinot : Corbieu ! Je suis bien coquillart / D'estre ainsi durement mené. »

(15.c) Mimin, v.49 : «par le[s] peulx de ma cotelle (...)»

On ne peut manquer de relever l'aspect ludique et la virtuosité déployée par les auteurs des farces, qui exercent leur créativité et font des jurons un ressort comique lié au monde à l'envers du carnavalesque ; le jeu avec le feu est excitant et permet des morceaux de bravoure, exactement comme les insultes. Les représentations de l'oralité dans le dialogue théâtral passent par le lexique de l'obscène et les interactions extrêmes. Les jurons compensent en quelque sorte la contrainte du vers, qui a tendance à amoindrir l'effet de réel produit par les choix registraux : le dialogue semble plus spontané, comme pris sur le vif.

Dans l'ensemble du corpus, au fil des siècles, les représentations des jurons dans le discours des personnages augmentent et se diversifient en littérature, tout comme la créativité des auteurs, contrairement au corpus didactique (dont la durée ne couvre, rappelons-le, que vingt ans, ce qui en restreint fortement la portée diachronique), et les Manieres de 1396 et 1399 rendent compte de cette évolution, mais celle de 1415, plus châtiée, montre aussi que l'euphémie, absente des deux précédentes, est en train de se mettre en place, même chez les locuteurs médians ; cette euphémie croissante est vraisemblablement liée à la répression dans la société et aux usages des locuteurs. L'évolution diachronique explique sûrement pourquoi les termes sont aussi variés d'une farce à l'autre, avec une surenchère dans la création de noms de saints fantaisistes, ou la référence à des parties du corps appartenant à l'indécence et au carnavalesque (comme avec le séant de la Vierge), par exemple. Cependant, les fabliaux sont déjà riches et le Roman de Renart présente très tôt des cas d'euphémie ${ }^{19}$. La variation diaphasique est donc certainement un critère plus important que la seule répression afin d'apprécier la variété des items, et une étude exhaustive des jurons littéraires mériterait d'être menée afin de rendre compte pleinement de la diachronie du phénomène. Cela dit, même si les Manieres sont moins hautes en couleurs que les fabliaux en

décomptes comme telle, puisque les items concernés sont déjà inclus aux autres catégories.

19 Voir ainsi dans le Roman de Renart, br. x, v.2715 : «Par la manbrance, / par les plaies, par la mort bieu, / ne sai ou sui ne en quel leu./» 
termes de créativité lexicale (et là n'est pas l'objectif d'un didacticien, recherchant par principe à montrer des usages courants), on y retrouve les catégories classiques de la littérature. L'usage des locuteurs doit donc se situer dans cet entre-deux.

\subsection{Quels usages ? Quels registres ? Quels actes?}

À partir de ces observations, se pose maintenant la question des usages des jurons et des actes qu'ils accomplissent. Y a-t-il des catégories lexicales plus blasphématoires ou offensives que d'autres? Quels locuteurs les emploient?

On notera tout d'abord dans les dialogues la grande fréquence de ces formules, qu'elles soient assertives ou expressives. Ces ponctuants du discours sont des sortes de «tics » du locuteur, qui ne semble même plus les contrôler tant ils sont présents et l'exemple (5.b) montrait par le métadiscours de L2 que la force illocutoire était bien calculée comme telle. Toutefois, on voit aussi dans cet exemple s'exercer le calcul de la portée illocutoire des énoncés par le tiers jugeant, lorsqu'il emploie « jurer » (cf. aussi 10.a) : même si les SN sont pris comme expressifs ou assertifs, ils peuvent choquer, comme en témoigne d'ailleurs la parcimonie de la Maniere de 1415 en la matière.

Nous avons déjà souligné lors du classement lexical les usages assertifs ou expressifs de ces SN : le test de commutation permet en effet d'en vérifier la portée pragmatique ; ainsi, dans l'exemple (6.a), où chaque prise de parole des deux locuteurs s'agrémente d'un juron, il serait possible de les remplacer par «sincèrement ", même si pour la dernière, une interjection pourrait aussi faire l'affaire (par exemple, « hau ! »). Cette troisième formule, juron expressif, bien que plus transgressive du fait de la référence à l'Ennemi, marque l'étonnement et la sympathie, soit des émotions et non un métadiscours sur la valeur du dire, comme avec les jurons assertifs.

On retrouve fréquemment ce mécanisme, là aussi partagé par les deux locuteurs, mais cette fois un homme et une femme, et l'on notera au passage l'abondance des formules référant à la divinité, tant pour souhaiter du bien à autrui que juste pour renforcer un énoncé :

(16) Man.1396, p.12 : «Et puis va la dame de l'hostel oveques le vadlet devers la chambre du seignour, et quant ele serra entré la chambre, elle dirra au seignour tout ainsi :

- Mon seignour, Dieu vous benoit et la compaignie.

- Si Dieux m'eide, ma tresdouce amye, il y a grant piece passé puis que je ne vous vei.

- Mays par m'anme, mon seignour, vous ditez veire.

-Par vostre foy, coment vous est il a jour de hui ?

- Tresbien, mon seignour, Dieu mercy et melx pur vous.

- Si Dieu m'eide, m'amye, j'en ay grant joie. Ore, m'amye, venez vous en, quar vous serrez ici devant moy en un chaier.

- Par Dieu, mon seignur, s'il vous plaist, non ferai.

- Par Dieu, si frés.

- Vostre merci, mon seignour. »

Chacun des jurons de la dame pourrait être remplacé par «sincèrement »; toutefois, ceux de l'homme sont moins nets, et s'apparentent plus à des marqueurs d'émotion voire d'intensité de l'expression, de l'insistance, comme le confirme le test de commutation. Les autres formules renvoyant à la déité sont elles aussi genrées, dans la mesure où celles de la dame s'apparentent à des formules de politesse (bienvenue, souhaits de bonne santé), tandis que celles de l'homme rejoignent son usage des jurons et pourraient tout à fait être supprimées sans que l'acte de langage accompli par l'énoncé ne change.

Notons que les jurons sont assez rares chez les locutrices des Manieres, ce qui corrobore de nombreuses descriptions du phénomène montrant que le juron est aussi un marqueur de virilité ou tout au moins de masculinité dans les corpus en diachronie (Hughes 1991 ; Vincent 1982 ; 
Lagorgette 2005b ; Gehweiler 2010). Les usages féminins seraient donc globalement plus proches de jurer1, dans le sens où ils engagent le locuteur et garantissent la vérité de l'énoncé ou la sincérité du locuteur. La Maniere de 1396 présente 5 occurrences sur un ensemble de $50(10 \%)^{20}$, celle de 1399 en présente 6 sur 33, soit $18 \%^{21}$, et celle de 14151 sur 9 , soit $11 \%^{22}$. On remarque, comme pour les insultes (Lagorgette 2013), que la Maniere de 1399, plus courte que celle de 1396, concentre les items transgressifs. On voit aussi que les deux catégories de jurons des dames réfèrent à elles-mêmes ou à la divinité, avec un cas d'euphémie dans la très sage et pieuse Maniere de 1415.

Le point commun à tous ces jurons est qu'ils interviennent dans des contextes non violents. Si nous nous penchons maintenant sur les jurons comme en (7), contenus dans les interactions verbales conflictuelles, où les émotions sont plus vives et où l'on pourrait s'attendre à des usages blasphématoires du fait d'un ressenti d'injustice face au sort, par exemple, nous remarquons que les jurons interviennent en complément d'autres actes de langage violents, tels que reproche, insulte et malédiction, mais aussi d'actes de langage où la divinité est invoquée pour son aide :

(17.a) Man. 1396, p.10 : «- Hé, Janyn, estez vous la?

- Oil dea, ne me poes tu veier? Quoi ne m'as tu, paillart, respondu a la primer parole que je t'appelloi ? Vel sic : Quoi ne me respondez vous a primer foiz que je hurtai a port ? Je pri a Dieu que il te poet mescheoir de cors, quar tu m'as fait longtens ici attendre et targer, et tu sais bien q'il ne fuit si grant froid cest anee com il fait a present, quar il negee, gresille et gelee si fort qe l'eue est si fort et espessee gelee com la laeure de mon pee, pur quoi je sai bien que la glaas du gelee ne se degelera en grant piece. Et pur ce overes la port tost et lesse moi entrer ciens, ou autrement je depesserai trestout, par la foi que je doi a Dieu, me croiez se tu vuis. »

Dans cette séquence de reproches et de menaces, exposée dans deux styles distincts (de part et d'autre de Vel sic qui articule les deux façons de construire ces actes de langage proposées), la formule «par la foi que je doi a Dieu » vient préciser combien les menaces sont sérieuses, et cette locution pourrait être paraphrasée par « sincèrement », et même par « je te garantis ». « Me croiez se tu vuis » renforce encore cette insistance sur la promesse d'action concrète comme devant être prise au sérieux.

Un autre dialogue fait ressortir de manière plus nette encore cette même dynamique : on y assiste à une querelle entre un maître et son subalterne qui non seulement, comme (17.a), renvoie à une faute, mais qui de plus met en scène des jugements moraux de l'un sur l'attitude de l'autre :

(17.b) Man.1396, p.19 : «Venez a moy. Ou si: Vien avant donqs.

- Maister, je vien a vous ore.

- Ou as tu eté depuis que tu levas? J'eusse esté au marché pieça se tu ne feus. Et tu sais bien que j'en fuy bien matyne levé pur aler au marché a viendre mes denrés. Male sepmaigne soit toy mys, car j'en ay perdu huy mon marché a cause de ta folie!

- Qu'en puisse je? Vous savez bien que j'ay esté ocupié entour vous bosignes, sicom moi comandastez hier soir.

- Hé, tu mens fausement. Tu as esté ovec tes filletz putaignes, et pur ce je pri a Dieu que tu puisse avoir le vit coupé, car je sçai bien que tu en aras male estraine a darrains se tu ne v[u]is lesse[r] ta folie, par Dieu !

- Mon seignour, je ne fus pas.

- Tu mens fausement parmy la gorge. Je sçai bien que tu y fus.

- Save vostre grace, non fas.

20 «Par m'anme » (p.12), «par Dieu» $($ pp.12, 13, 41), «par ma foy » (p.27).

21 «Par Dieu », p.57; «par ma alme », p.60, p.64; «par ma foy que mien est », p.61 / «par ma foy», p.62; « par Saint Jaques », p.63.

«Par Diee », p.75. 
- Teis toy, de par le deable. Ou si: Finés vous. Ou si: Tien te coy ou te je doneray un ytiel sufflet que tu penseras de moy decy as quatre jours, me croyez se tu vis, car je teneray covenant. Ou si: Tenés vous coy. Ou si: Finés vous. Ou si: ne me parlés ja plus a ceste foitz sur peril qu'en purra avenir, quar se vous le frés, vous en arés dez horions si bien assés que par aventure vous penseras de moy decy as troys sepmaignes, me croiés se vous voillés, quar vraiment je vous tiendray la covenant.

- Hé, mon tresdoulx maister, je vous cri mercy et vous en supplie humblement de vostre grace que vous me voillés pardonere vostre maletalent, quar s'il Dieu pleist, je ne mesprenderay ja plus envers vous.

- Ore va t'en, quar je te pardone.

- Grant mercy, mon seignour. »

Cette séquence fort bien bâtie du point de vue didactique permet de montrer comment formuler argumentaire, malédictions (Male sepmaigne soit toy mys ; je pri a Dieu que tu puisse avoir le vit coupé), ordres (section à l'impératif), menaces (quar se vous le frés...la covenant), excuses (je vous cri mercy...vostre maletalent), promesse (quar s'il Dieu pleist, je ne mesprenderay ja plus envers vous), pardon (Ore va t'en, quar je te pardone). Les seuls jurons dans cet ensemble viennent du maître et marquent son ire : par Dieu clôt l'exposé des raisons de sa colère et de sa malédiction, tandis que de par le deable accompagne un ordre, celui de se taire car L2 persiste à mentir alors que L1 est persuadé d'avoir raison. Leur appartenance au blasphème semble peu pertinente ; en revanche, ils accompagnent d'autres male dicta et prennent bien la divinité à témoin, dans le premier cas, ou invoquent celui qui finira par hériter de l'âme du mécréant châtié s'il persiste en ses errances.

On notera, dans ce même exemple, que de par le deable, présenté en premier dans la liste des possibilités de formuler l'ordre de se taire, est associé au tutoiement et semble être le modèle non pas standard (car la référence au diable surgit surtout dans les échanges violents) mais médian, tandis que la dernière formulation, petit exercice de style, semble plus littéraire et soutenue. Cela dit, les textes littéraires, et en particulier les farces, mais aussi les récits brefs, choisissent plutôt la version médiane. Enfin, si le lexique des male dicta peut référer de manière explicite au corps grotesque, on ne trouve jamais de juron de ce type, alors que fabliaux et farces en regorgent : le tabou est donc maintenu par le professeur moraliste.

\section{CONCLUSION : LE DISCOURS TRANSGRESSIF ET SA FORCE}

À partir des différents corpus que nous venons d'examiner, il semble que les jurons peuvent intervenir tant dans un oral relâché que dans un écrit plus soutenu ; toutefois, ce ne sont pas les mêmes lexèmes qui rempliront ces différents rôles. Globalement, les références à Dieu ou à soimême seraient du côté de l'acceptable, tandis que les références au diable seraient limitées aux interactions violentes. Toutefois, selon les choix lexicaux des locuteurs, les deux catégories «modérées » peuvent aussi devenir plus transgressives lorsqu'elles renvoient à des parties du corps taboues, qui les font passer dans des registres relâchés, et l'on notera que le genrage des jurons est bien établi dans les Manieres de langage : soit le texte permet aux dames le juron, mais il reste « décent » (Manieres de 1396 et 1399), soit il est tout bonnement exclu en soi (Maniere de 1415). Les publics auxquels s'adressent les textes orientent le choix du professeur : aux voyageurs marchands, aux artisans la langue populaire, aux autres un registre plus médian. S'ajoute à ce paramètre celui du lieu de production, qui est présenté comme jouant aussi sur les choix lexicaux. En résumé, les Manieres, chacune à leur façon, mettent en scène le juron comme participant de l'oralité spontanée des locuteurs et comme marqueur typique du français parlé ; c'est donc un indicateur culturel témoignant aussi du genre, de la classe sociale d'appartenance et de la zone géographique d'origine. Ces manuels rejoignent en cela les textes littéraires où les représentations de l'oralité sont fondamentales dans la dynamique de l'œuvre, qu'il s'agisse des dialogues des 
fabliaux ou du théâtre, et dans le stéréotypage des personnages. Proposant différents registres pour l'expression des émotions, selon leur intensité, les Manieres montrent le juron dans deux de ses trois dimensions : d'une part, l'assertion de la sincérité du locuteur, et d'autre part, l'expression d'un fort ressenti ; toutefois, contrairement aux textes littéraires, elles ne recourent jamais dans le juron à des termes choquant les bonnes mœurs. Elles mettent aussi en relief leur caractère mécanique et désémantisé : point de vilain serement dans ces textes. Mais les jurons à valeur pragmatique assertive ou expressive sont naturellement présents lorsque des actes de langage de type male dicta sont produits, respectant ce que révèlent les registres criminels ou autres actes juridiques. Le discours transgressif forme donc un tout, dont le juron, comme l'insulte, peut être un marqueur privilégié ; toutefois, le point le plus important est que très rares, voire absents sont les blasphèmes, au sens strict, alors que nombreuses sont les occurrences. C'est donc très tôt que le tabou linguistique a fonctionné à l'écrit, d'abord en gommant les jurons des textes, puis en n'en montrant que la face désémantisée. Marqueur d'oralité spontanée par excellence, comme l'interjection et le terme d'adresse, le juron est donc un ressort de vraisemblance non négligeable, même s'il peut sentir le souffre.

\section{CORPUS :}

Choix de fabliaux, Guy Raynaud de Lage éd., Paris, Champion, 1986.

Eighteen Anglo-Norman Fabliaux, ian Short et R. Pearcy éd., London, Anglo-norman Text Society, 2000 ; en ligne sur : http://www.anglo-norman.net/cgi-bin/and-getloc

Fabliaux, Rosanna Brusegan éd., Paris, 10/18, 1994.

Fabliaux érotiques, Luciano Rossi éd., Paris, LGF, Lettres Gothiques, 1992.

Manières de langage de 1396, 1399 et 1415, Andres M. Kristol éd., London, Anglo-norman Text Society (from Birkbeck College), 1995.

Recueil de farces (1450-1550), André Tissier éd., Genève, Droz : t.III (Le Cuvier, Le Chaudronnier, Le Pâté et la tarte, Jenin fils de rien), 1988, t.iV (Le Ramoneur de cheminées, Le Meunier de qui le diable emporte l'âme en enfer), 1989, t.VII (La Farce de Maistre Pathelin), 1993.

Selected fabliaux, Brian Joseph Levy éd., Department of French, Hull, 1978.

\section{Bibliographie :}

Austin, J. L., 1970, Quand dire c'est faire, Paris, Le Seuil.

Benveniste, E., 1974, « Blasphémie et euphémie », Problèmes de linguistique générale, t.2, Paris, Gallimard, p. 254-257.

Cabantous, A., 1998, Histoire du blasphème en Occident, Paris, Albin Michel.

Casagrande, C., Vecchio, S., 1991, Les péchés de la langue. Discipline et éthique de la parole dans la culture médiévale, trad. de l'italien par Ph. Baillet, Paris, Cerf.

Favret-Saada, J., 1977, Les Mots, la mort, les sorts, Paris, Gallimard.

Gauvard, C., 1991, « Le crime et le sacré. Tuer Dieu et soi-même », dans EAD., « De grace especial ». Crime, État et société en France à la fin du Moyen Age, Paris, Publications de la Sorbonne, t. II.

Gehweiler, E., 2010, « Interjections and expletives », Historical Pragmatics, A. H. Jucker and I. Taavitsainen (eds), p. 315-349.

Hughes, G., 1991, A Social History of Foul Language, Oaths and Profanity in English, Oxford, Blackwell.

Huston, N., 1980, Dire et interdire, Paris, Payot.

Jucker, A. H., Taavitsainen, I., 2000, « Diachronic speech act analysis: insults from flyting to flaming », Journal of Historical Pragmatics, 1-1, p. 67-95.

Lagorgette, D., 1998, Désignatifs et termes d'adresse dans quelques textes en moyen français, Université Paris 10-Nanterre, thèse dactylographiée.

Lagorgette, D., 2003, « Les syntagmes nominaux d'insulte et de blasphème : analyse diachronique du discours 
marginalisé », Thélème, Madrid, Univ. Complutense, p. 171-188.

Lagorgette, D., 2005a, « La vérité du nom : métadiscours sur le droit nom, métadiscours sur l'origine? «, Bien dire, Bien aprandre, 23, Lille, P.U. de Lille 3, p. 277-293.

Lagorgette, D., 2005b, «Jouer avec le feu : euphémie et blasphémie en diachronie », Le Jeu, J. Derive et S. Santi éds., Université de Savoie, p. 169-200.

Lagorgette, D., 2013, «Insultes et registres de langue dans les Manières de langage : transgression et effets d'oralité », Diachroniques 3, p. 119-147.

Lauret, B., 1993, «Tu ne prononceras pas le nom de Dieu en vain », Blasphèmes et libertés, dir. par Dartevelle, P., Denis, Ph. et Robyn, J., Paris, Ed. du Cerf, p. 33-54.

Leveleux-Texeira, C., 2000, « Dire et interdire. Le discours juridique entre omission et action. L'exemple du blasphème (XII $-\mathrm{XVI}^{\mathrm{e}}$ siècles) », Cahiers de recherches médiévales, 7, http://crm.revues.org/document885.html

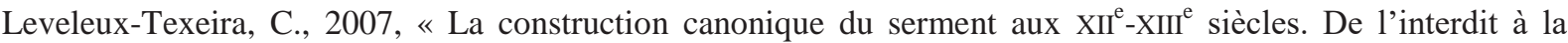
norme ", Comptes rendus des séances de l'Académie des inscriptions et Belles-Lettres 151-2 ; p. 821-844. http://www.persee.fr/web/revues/home/prescript/article/crai_0065-0536_2007_num_151_2_87953

Leveleux-Texeira, C., 2008, «Injure à Dieu, outrage au roi. Le blasphème à la fin du moyen âge et au début de l'époque moderne : un crime limite », dans Outrages, insultes, blasphèmes et injures : violences du langage et polices du discours, E. Desmons et M.-A. Paveau (éds.), Paris, L’Harmattan, 2008, p. 31-51.

Leveleux-Texeira, C., 2011, «Entre droit et religion : le blasphème, du péché de la langue au crime sans victime », Revue de l'histoire des religions, 4, p. 587-602 ; http://rhr. revues.org/7828

Lodge R. A., 1997, Le Français, histoire d'un dialecte devenu langue, Paris, Fayard.

Orr, J., 1957, « De l'étymologie des jurons », Cahiers de l'Association internationale des études françaises 9 , p. 278-286.

Payen, J.-C., 1968, Le Motif du repentir dans la littérature médiévale, des origines à 1230, Genève, Droz.

Pichette, J.-P., 1980, Le Guide raisonné des jurons, Montréal, Quinze.

Taavitsainen, I., 1997a, «Exclamations in Late Middle English », Studies in Middle English Linguistics. J. Fisiak (ed), Berlin and new York, Mouton de Gruyter, p. 573-607.

Taavitsainen, I., 1997b, « By Saint Tanne: Pious Oaths or Swearing in Late Middle English », dans Festschrift for Jacek Fisiak. R. Hickey (ed), Berlin and new York, Mouton de Gruyter, p. 815-26.

Vincent, D., 1982, Pressions et impressions sur les sacres au Québec, Québec, Editeur officiel.

Vincent, D., 1993, Les ponctuants de la langue et autres mots du discours, Québec, Nuit blanche.

Wierzbicka, A., 2002, «Australian cultural scripts - bloody revisited », Journal of Pragmatics, 34 ; p. 1167 1209.

Zumthor, P., 1953, «Euphémisme et rhétorique au Moyen Age », Cahiers de l'Association internationale des études francaises, 3-5, p. 177-184. 